\title{
Mối quan hệ giữa chia sẻ tri thức, sự hài lòng trong công việc và hiệu suất làm việc: Trường hợp các công ty khởi nghiệp trong lĩnh vụcc du lịch trên địa bàn Thành phố Hồ Chí Minh \\ The relationship between knowledge sharing, job satisfaction and work performance: The case study of tourism startups in Ho Chi Minh City
}

\author{
Nguyễn Hữu Nghi $1^{1 *}$, Mai Trường $\mathrm{An}^{1}$ \\ ${ }^{1}$ Trường Đại học Quốc tế Hồng Bàng, Việt Nam \\ "Tác giả liên hệ, Email: huunghisg@ gmail.com
}

\section{THÔNG TIN}

DOI:10.46223/HCMCOUJS. econ.vi.13.2.1612.2018

Ngày nhận: 03/10/2017

Ngày nhận lại: 06/02/2018

Duyệt đăng: 14/03/2018

Tù khóa:

chia sẻ tri thức, doanh nghiệp khởi nghiệp, hiệu suất làm việc, lĩnh vực du lịch, sự hài lòng trong công việc

Keywords:

job satisfaction, knowledge sharing, SCT, startup, TPB, TRA, tourism industry, work performance

\section{TÓM TÁT}

Nghiên cứu được thực hiện nhằm kiểm định mối quan hệ giữa việc chia sẻ tri thức (Knowledge Sharing - KS), sự hài lòng trong công việc (Job Satisfaction - JS) và hiệu suất làm việc (Work Performance - WP) của nhân viên của các công ty khởi nghiệp trong lĩnh vực du lịch trên địa bàn Thành phố Hồ Chí Minh. Thông qua khảo sát 322 nhân viên, dữ liệu thu thập được xử lý bằng phần mềm SPSS 23.0 và AMOS 20.0. Kết quả nghiên cứu cho thấy việc thu nhận tri thức (Knowledge Collecting - KC) và cho tặng tri thức (Knowledge Donating $\mathrm{KD})$ đều có ảnh hưởng tích cực đến sự hài lòng và hiệu suất làm việc của nhân viên. Trong đó thu nhận tri thức có tác động mạnh hơn cho tặng tri thức.

ABSTRACT
The research aims at investigating the relationship between
Knowledge Sharing (KS), Job Satisfaction (JS) and Work
Performance (WP). The research data was collected from 322
employees of tourism startups in Ho Chi Minh City. It was
analyzed by SPSS 23.0 and AMOS 20.0. The study results
reveal that both employees' Knowledge Donating (KD) and
Knowledge Collecting (KC) have a major impact on their
satisfaction and performance. In fact, knowledge collecting has
a stronger impact than knowledge donating.




\section{1. Đặt vấn đề}

Để tồn tại, phát triển và bảo toàn những lợi thế cạnh tranh của doanh nghiệp là thách thức cho bất cứ nhà quản trị nào. Nhằm đạt được những mục tiêu đó, nhà quản trị không những cần phải biết khai thác một cách hiệu quả các nguồn lực hữu hình hiện có của tổ chức, mà còn phải biết phát triển các nguồn lực vô hình đáp ứng nhu cầu phát triển của tổ chức. Một trong những nguồn lực vô hình mà bài viết này đề cập đó là nguồn lực tri thức. Tri thức đóng vai trò cực kỳ quan trọng trong kinh doanh (du Plessis, 2007) và là một loại nguồn lực giúp cho doanh nghiệp tăng trưởng (Business Link, 2009) trong môi trường kinh doanh nhiều biến động hiện nay. Trong một tổ chức kinh doanh, tri thức là yếu tố quan trọng không những làm gia tăng khả năng sáng tạo mà còn củng cố lợi thế cạnh tranh bền vững cho tổ chức (Alegre, Sengupta, \& Lapiedra, 2013).

Tri thức là thông tin được sử dụng và chia sẻ để đạt được các mục tiêu của tổ chức ${ }^{1}$, nó được tạo ra và luân chuyển giữa các thành viên trong tổ chức thông qua những bài học kinh nghiệm và thực tiễn tốt nhất. Sau đó những tri thức này sẽ được lưu trữ theo những cách tốt nhất để có thể thu hồi cũng như tái sử dụng dễ dàng trong tương lai đáp ứng nhu cầu phát triển của tổ chức (Dalkir, 2007). Nguồn tài sản tri thức này được hình thành thông qua việc tạo và phổ biến những kiến thức từ các thành viên trong tổ chức được kết tinh và liên kết với nhau thông qua hệ thống quản lý tri thức của tổ chức (Nonaka, von Krogh, \& Voelpel, 2006).

Theo một số cuộc hội thảo khoa học và diễn đàn về khởi nghiệp thì có khoảng $80 \%$ các khởi nghiệp bị thất bại (Doanh nhân Sài Gòn, 2017), một trong số những nguyên nhân đó là do không tập hợp được đội ngũ phù hợp từ đó dẫn đến thiếu động cơ, kiến thức chuyên môn hoặc tầm nhìn chung (Lance, 2016) để tạo sự liên kết và chia sẻ kiến thức và kinh nghiệm cần thiết (Tam Mai, 2017).

Du lịch là lĩnh vực kinh tế với đặc trưng các mô hình kinh doanh có qui mô nhỏ và vừa chiếm ưu thế nên được xem là một lĩnh vực rất có tiềm năng để khởi nghiệp như tour khám phá, du lịch homestay, hàng thủ công mỹ nghệ... là những thành tố luôn sẵn có và rất dễ thực hiện các dự án khởi nghiệp. Trong lĩnh vực du lịch, kỹ năng tương tác giữa nhân viên với khách hàng và kiến thức nghề nghiệp giữ vai trò quyết định việc sự thành bại trong kinh doanh. Nhưng du lịch là một ngành có tỉ lệ chuyển đổi nhân lực (turnover) rất cao, nên khi một nhân viên ra đi họ mang theo cả kinh nghiệm, kiến thức, bí quyết, các mối quan hệ với khách hàng, ... Do đó việc chia sẻ những kỹ năng và kiến thức được xem là một tiến trình quan trọng trong việc quản lý tri thức (Nooshinfard \& Nemati-Anaraki, 2014). Vì vậy, làm sao để các thành viên trong tổ chức, nhất là trong giai đoạn khởi sự nhận được sự động viên cần thiết để hình thành văn hóa chia sẻ kinh nghiệm và kiến thức của mỗi người thành tri thức chung là vô cùng quan trọng giúp cho tổ chức có được năng lực cần thiết để tồn tại và phát triển. Đây cũng là lý do chính mà bài nghiên cứu này hướng đến.

\section{Cơ sở lý thuyết và mô hình nghiên cứu}

\subsection{Co sở lý thuyết}

\section{Khái niệm chia sẻ tri thức}

\footnotetext{
${ }^{1}$ http://isoconsultantpune.com/iso-90012015-organizational-knowledge/
} 
Hooff và Ridder (2004) định nghĩa chia sẻ tri thức là một quá trình mà các cá nhân trao đổi tri thức tiềm ẩn và tri thức hiện hữu để tạo ra tri thức mới. Theo Lin (2007), chia sẻ tri thức có thể được định nghĩa như một văn hóa tương tác mang tính xã hội, liên quan đến việc nhân viên của các bộ phận, tổ chức trao đổi kiến thức, kinh nghiệm và kỹ năng. Ví dụ như nhân viên sẵn lòng giao tiếp, chia sẻ kiến thức với đồng nghiệp và học hỏi lẫn nhau. Cũng theo Gao, Li, và Clarke (2008), chia sẻ tri thức là quá trình cho và nhận tri thức, trong đó sự sáng tạo và chia sẻ tri thức phụ thuộc vào nỗ lực có ý thức của cá nhân làm cho tri thức được chia sẻ. Theo Hooff và Ridder (2004), cho tặng tri thức là việc một cá nhân giao tiếp với người khác về vốn trí tuệ của mình, thu nhận tri thức là việc một cá nhân hỏi ý kiến đồng nghiệp để tiếp thu vốn trí tuệ của họ. Liao, To, và Hsu (2013) cũng định nghĩa chia sẻ tri thức như một sự chia sẻ cộng đồng liên quan đến thông tin, ý tưởng, những gợi ý hay những kiến thức chuyên môn của mỗi người. Như vậy, có thể hiểu chia sẻ tri thức là quá trình cho và nhận tri thức, làm cho tri thức có thể được tái sử dụng bởi những người khác.

\section{Khái niệm sụ hài lòng trong công việc}

Hoppock (1935) là một trong những người đầu tiên đưa ra khái niệm về sự hài lòng trong công việc. Ông cho rằng sự hài lòng trong công việc là một khái niệm tổng thể; vì vậy, cách đơn giản nhất để đo lường sự hài lòng là xem xét mức độ đánh giá tổng thể của nhân viên đối với công việc. Adams (1963) thì xem xét sự hài lòng trong công việc theo khía cạnh công bằng. Ông gợi ý rằng, khi một người nhận thấy những gì mình nhận được từ công việc công bằng với trình độ và năng lực của mình thì họ sẽ hài lòng hơn với công việc. Mặt khác, Porter và Lawler (1968) cho rằng sự hài lòng được tạo ra khi nhu cầu và ham muốn được đáp ứng. Theo Weiss (2002), mức độ sự hài lòng trong công việc được định nghĩa như là một thái độ làm việc tích cực hay tiêu cực của người lao động đối với công việc của họ. Tóm lại, sự hài lòng trong công việc là cảm giác hài lòng của nhân viên khi cảm nhận công sức mình bỏ ra xứng đáng với kết quả mình nhận được và sự hài lòng trong công việc được đánh giá thông qua sự cố gắng và thành tích làm việc.

\section{Khái niệm hiệu suất làm việc}

Hall và Goodale (1986) chỉ ra rằng hiệu suất làm việc là cách một nhân viên thực hiện nhiệm vụ của mình thông qua việc sử dụng thời gian, kỹ thuật và tương tác với các cá nhân khác. Theo Schermerhorn (1989), hiệu suất làm việc được hiểu là số lượng và chất lượng công việc đạt được bởi một cá nhân hay một nhóm. Trong bài nghiên cứu này, hiệu suất làm việc được hiểu là hiệu suất công việc của cá nhân đang làm việc trong các công ty khởi nghiệp trong lĩnh vực du lịch trên địa bàn Thành phố Hồ Chí Minh.

\section{Mối quan hệ giũa chia sẻ tri thức, sụ hài lòng trong công việc và hiệu suất làm việc}

Hooff và Ridder (2004) trong công trình nghiên cứu của mình đã chỉ ra rằng việc chi sẻ tri thức là một tiến trình bao gồm việc cho, tặng tri thức (knowledge donating) và thu nhận tri thức (knowledge collecting); và chia sẻ tri thức cũng có tác động tích cực đến sự hài lòng của một cá nhân trong tổ chức. Tương tự, Teh và Sun (2012) cũng chứng minh được rằng chia sẻ tri thức có tác động tích cực đến sự hài lòng của nhân viên. Trong thời đại mà kiến thức trở thành lợi thế cạnh tranh của một doanh nghiệp nói chung hay một cá nhân nói riêng thì việc chia sẻ tri thức để học hỏi và nâng cao trình độ sẽ giúp cá nhân đó hài lòng hơn với công việc. 
Thực tế cho thấy, có rất nhiều cá nhân gia nhập vào một công ty hay tổ chức nào đó không phải đơn giản chỉ vì mức lương được đề nghị mà là vì sự dự đoán và mong muốn về những kiến thức, kinh nghiệm lẫn trải nghiệm họ sẽ có được, và xa hơn nữa là những tri thức họ thu được sẽ là tiền đề cho mức thu nhập kỳ vọng trong tương lai.

Bên cạnh đó tác động đến sự hài lòng của một cá nhân trong tổ chức, chia sẻ tri thức cũng có tác động đến hiệu suất làm việc của cá nhân đó (Aksoy, Ayranci, \& Gozukara, 2016; Henttonen, Kianto, \& Ritala, 2016; Saeed, 2016). Nghiên cứu của Bontis, Richards, và Serenko (2011) cũng chỉ ra rằng nhân viên tìm kiếm và chia sẻ thông tin để hoàn thành nhiệm vụ và để đáp ứng nhu cầu cá nhân; điều đó có nghĩa là họ sẽ tích cực tham gia vào các hoạt động của tổ chức và đạt được mục tiêu cao hơn khi họ chia sẻ tri thức và hài lòng với công việc. Theo Weiss (2002), sự hài lòng sẽ là động lực để nhân viên phấn đấu hoàn thành nhiệm vụ được giao. Vì vậy, chia sẻ tri thức cũng có tác động đến hiệu quả làm việc của nhân viên trong một tổ chức và sự hài lòng cũng có tác động đến hiệu suất làm việc của họ.

\subsection{Các lý thuyết nền và Mô hình nghiên cứu đề xuất}

Thuyết hành động hợp lý (Theory of Reasoned Action - TRA) và thuyết hành vi dự định - The theory of planned behavior - TPB (Madden, Ellen, \& Ajzen, 1992) được nhiều nhà nghiên cứu sử dụng để kiểm định mối quan hệ giữa hành vi dự định và các nhân tố khác như niềm tin, các chuẩn giá trị ...Chow và Chan (2008) và Orces và Kim (2005) đã sử dụng Thuyết hành động hợp lý (Theory of Reasoned Action - TRA) để nghiên cứu về những giá trị xã hội và những lý do tại sao mọi người trong tổ chức lại sẵn sàng hoặc không sẵn sàng chia sẻ tri thức. Việc chia sẻ tri thức không thể có hiệu quả nếu bị ép hay bắt buộc mà quá trình này phải thông qua nỗ lực tạo ra môi trường làm viêc thoải mái, tạo ra các động lực thúc đẩy đến các cá nhân thông qua việc kiểm định mối quan hệ giữa mạng xã hội, niềm tin và chia sẻ mục tiêu đến dự định chia sẻ tri thức thông qua biến trung gian thái độ và tiêu chuẩn chủ quan trên cơ sở mẫu chỉ là các quản lý (managers) từ các tổ chức và nghiên cứu hành vi chia sẻ kiến thức trong địa giới Hong Kong để kiểm định ý định sẵn sàng hay không sẵn sàng chia sẻ kiến thức. Trong khi đó, Orces và Kim (2005) chứng tỏ trong nghiên cứu của mình rằng khi nhân viên được thúc đẩy và hình thành nhu cầu hiểu biết thì khi đó nhu cầu này trở thành động lực căn bản hình thành hành vi chia sẻ kiến thức qua việc hội nhập Thuyết hành vi dự định - The theory of planned behavior - TPB trên cơ sở xem xét mối quan hệ động lực bên trong và động lực bên ngoài tác động như thế nào đến thái độ và dự định chia sẻ tri thức dựa trên dữ liệu theo mặt cắt dọc từ trên xuống dưới chứ không trải theo bề rộng, các mối quan hệ nhân quả được suy luận thay vì được minh chứng. Như vậy cần phải thực hiện các nghiên cứu khác với mẫu là đội ngũ nhân viên có sẵn sàng chia sẻ tri thức với nhau hay không, bao gồm tri thức tổng quát và tri thức chuyên ngành cụ thể như du lịch trong những bối cảnh khác nhau về văn hóa quốc gia (Orces \& Kim, 2005).

Trong nghiên cứu của mình, Chiu, Hsu, và Wang (2006) lại chỉ ra rằng kết quả kỳ vọng của cộng đồng đóng vai trò quan trọng đến số lượng và chất lượng kiến thức được chia sẻ, trong khi những kết quả mong đợi của cá nhân lại ảnh hưởng tiêu cực nhưng không đáng kể đến số lượng kiến thức được chia sẻ thông qua việc sử dụng 2 thuyết "Nhận thức xã hội và Tài nguyên xã hội - Social Cognitive Theory and the Social Capital Theory” - nhằm khám phá động cơ và 
kỳ vọng cá nhân đằng sau việc chia sẻ tri thức trong các cộng đồng ảo. Tương tự như 2 nghiên cứu đã đề cập ở trên nghiên cứu của Chiu và cộng sự cùng một cách tiếp cận dữ liệu theo mặt cắt từ trên xuống dưới và chỉ tập trung vào một khía cạnh là người trao mà chưa khám phá khía cạnh người nhận tri thức, trong khi việc chia sẻ tri thức phải hội đủ 2 chiều thì mới là yếu tố được xem là bền vững của hệ thống quản lý. Vì vậy, cần có những nghiên cứu sâu hơn để có một sự hiểu biết hơn về mối tương quan giữa các khía cạnh của nguồn vốn tri thức (Chiu et al., 2006).

Hai tác giả Suliman và Hosani đã thực hiện một cuộc nghiên cứu thực nghiệm vào năm 2014 để nghiên cứu tác động của sự hài lòng trong công việc đến chia sẻ tri thức. Dựa vào "Thuyết động lực tạo ra tri thức trong tổ chức" - Dynamic theory of organizational knowledge creation - và nhiều công trình nghiên cứu khác, Suliman và Hosani (2014) chứng minh được rằng chia sẻ tri thức là một tiến trình bao gồm việc cho và nhận tri thức. Đồng thời kết quả nghiên cứu cũng khẳng định sự tác động trực tiếp và tích cực của sự hài lòng trong công việc lên chia sẻ tri thức của nhân viên trong một tổ chức. Kết quả nghiên cứu dựa trên mẫu là nhân viên trong một tập đoàn (dầu khí) nên thực tế có thể không phản ánh đúng trong các ngành kinh tế khác. Do đó cần có những nghiên cứu khác tại các ngành khác trong khung địa giới khác để xác định rõ hơn bản chất mối quan hệ giữa sự hài lòng và hành vi chia sẻ tri thức trong tổ chức.

Ngược lại với nghiên cứu của Suliman và Hosani (2014), Trivellas, Akrivouli, Tsifora, và Tsoutsa (2015) đề xuất mô hình nghiên cứu gồm chia sẻ tri thức tác động đến năng lực tổng quát của nhân viên và sự hài lòng trong công việc. Theo Trivellas và cộng sự (2015), chia sẻ tri thức là yếu tố cốt lõi của quản trị tri thức, nó phản ánh sự sẵn lòng của nhân viên khi chia sẻ kinh nghiệm, kiến thức với người khác; đồng thời tạo điều kiện cho việc trao đổi thông tin có ích trong tổ chức thông qua việc kiểm định mối quan hệ giữa việc chia sẻ tri thức và sự hài lòng trong công việc thông qua sự tác động của biến điều tiết năng lực của các thành viên trong tổ chức. Dựa trên những giá trị được chia sẻ, các cá nhân sẽ ngày càng hoàn thiện bản thân và hài lòng hơn với công việc. Vì vậy việc chia sẻ tri thức sẽ có tác động tích cực đến sự hài lòng của nhân viên trong tổ chức. Trivellas và cộng sự (2015) đề xuất các nghiên cứu tương lai kiểm định lại kết quả thông qua các biến số như văn hóa tổ chức hay quốc gia tác động như thế nào đối với nhân viên có năng lực khác nhau và các biến số nội bộ như động cơ, phong cách lãnh đạo, trí tuệ cảm xúc và sự sáng tạo.

Mô hình nghiên cứu được đề xuất trên cơ sở các lý thuyết nền và các hướng đề xuất nghiên cứu của nghiên cứu đã đề cập bên trên, nhóm tác giả thấy rằng các công trình nghiên cứu đã đề cập tập trung vào xác định cách thức hình thành động cơ và hành vi chia sẻ tri thức giữa các cá nhân trong tổ chức. Trong ngành du lịch, đặc biệt các mô hình khởi nghiệp kinh doanh với qui mô nhỏ, nên việc chia sẻ tri thức sẽ là tiền đề quan trong nhằm gia tăng hiệu suất (Oyemomi, Liu, Neaga, \& Alkhuraiji, 2016) của tổ chức, không những vậy nó còn giúp tổ chức tạo và duy trì lợi thế cạnh tranh (Nooshinfard \& Nemati-Anaraki, 2014). Nhóm tác giả đề xuất mô hình nghiên cứu như sau: 


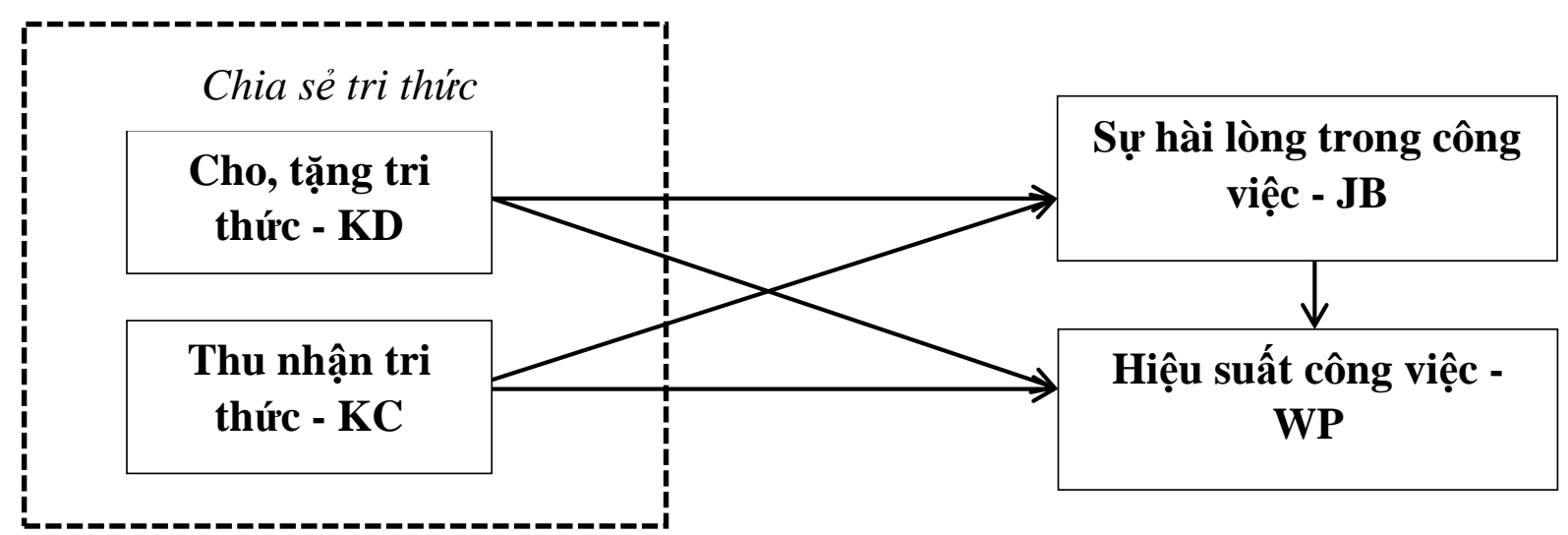

Hình 1. Mô hình nghiên cứu đề xuất

Nguồn: Nhóm tác giả (2017)

\section{Thiết kế nghiên cứu}

Đối tượng khảo sát là những nhân viên đang làm việc trong các công ty khởi nghiệp trong lĩnh vực du lịch với qui mô nhỏ và vừa (Chính phủ, 2009) (dựa theo số lượng lao động của công ty) trên địa bàn Thành phố Hồ Chí Minh, những công ty có thâm niên hoạt động được 5 năm tính đến thời điểm 06/2017. Dữ liệu được thu thập bằng cách phát bảng câu hỏi và được xử lý bằng phần mềm SPSS 23.0 và Amos 20.0.

Mô hình đo lường gồm 21 biến quan sát. Trong đó, thang đo cho tặng tri thức - KD gồm 7 biến quan sát và thang đo thu nhận tri thức - KC gồm 6 biến quan sát được kế thừa và phát triển từ thang đo của Hooff và Ridder (2004); thang đo sụ hài lòng trong công viẹc gồm 4 biến quan sát được kế thừa từ thang đo của Ollo-López, Bayo-Moriones, và Larraza-Kintana (2016); thang đo hiệu suất làm việc - WP gồm 4 biến quan sát được kế thừa và chỉnh sửa từ thang đo của Henttonen và cộng sự (2016).

350 bảng câu hỏi được gửi đến cho $275^{2}$ tổ chức/doanh nghiệp/công ty hoạt động trong lĩnh vực du lịch ${ }^{3}$ trên địa bàn thông qua đầu mối là Phòng Tổ chức - Hành chính, nhưng chỉ thu về được 337 bảng, trong đó có 15 bảng mà người được khảo sát không trả lời hết các câu hỏi hoặc đánh cùng một sự lựa chọn. Như vậy, chỉ có 322 bảng khảo sát hợp lệ được nhận từ 217 tổ chức/doanh nghiệp/công ty và đây cũng chính là kích thước mẫu chính thức của nghiên cứu này.

\footnotetext{
2300 doanh nghiệp này được lọc từ nguồn cung cấp khoảng 600 đơn vị từ Tổng đài 1080, danh bạ Hội viên của VCCI - CN thành phố Hồ Chí Minh và đối chiếu với số liệu công bố trên website của Sở Du lịch - Cơ quan quản lý về chuyên ngành

${ }^{3} \mathrm{http}: / /$ sodulich.hochiminhcity.gov.vn/web/guest/216
} 


\section{Kết quả nghiên cứu}

\section{1. Đánh giá độ tin cậy của thang đo bằng hệ số Cronbach's Alpha}

\section{Bảng 1}

Tổng hợp hệ số Cronbach's Alpha

\begin{tabular}{|l|c|c|c|}
\hline \multicolumn{1}{|c|}{ Thang đo } & $\begin{array}{c}\text { Số biến quan } \\
\text { sát }\end{array}$ & $\begin{array}{c}\text { Cronbach's } \\
\text { Alpha }\end{array}$ & $\begin{array}{c}\text { Hệ số tưong } \\
\text { quan biến } \\
\text { tổng } \\
\text { thấp nhất }\end{array}$ \\
\hline Cho, tặng tri thức - KD & 7 & 0.892 & 0.594 \\
\hline Thu nhận tri thức - KC & 6 & 0.876 & 0.371 \\
\hline Sự hài lòng trong công việc - JS & 4 & 0.880 & 0.605 \\
\hline Hiệu suất công việc - WP & 4 & 0.906 & 0.720 \\
\hline
\end{tabular}

Nguồn: Kết quả phân tích dữ liệu của nhóm tác giả (2017)

Kết quả kiểm định độ tin cậy thang đo bằng hệ số Cronbach's Alpha cho thấy các biến $\mathrm{KD}, \mathrm{KC}, \mathrm{JS}$ và WP có hệ số Cronbach's Alpha nằm trong khoảng [0.75 - 0.95] nên các thang đo được sử dụng trong bài đạt yêu cầu; tất cả các biến quan sát đều có tương quan biến tổng lớn hơn 0.3 nên tất cả các biến quan sát đều được giữ lại và đưa vào phân tích EFA.

\subsection{Phân tích nhân tố EFA}

Theo Gerbing và Anderson (1988), phương pháp trích Principal Axis Factoring với phép xoay Promax sẽ phản ánh cấu trúc dữ liệu chính xác hơn phương pháp trích Principal Components với phép xoay Varimax. Vì vậy để đảm bảo kết quả trong phân tích cấu trúc tuyến tính SEM tiếp theo, trong phân tích EFA cho nhóm biến $\mathrm{KD}, \mathrm{KC}$ và JS tác giả chọn phương pháp trích Principal Axis Factoring với phép xoay Promax. Phân tích EFA cho biến phụ thuộc vẫn sử dụng phép quay Varimax với phương pháp trích Principal Component.

\section{Kết quả phân tích EFA lần 1 cho các biến $K D, K C$ và $J S$}

Hệ số $\mathrm{KMO}=0.906>0.6$ nên EFA phù hợp với dữ liệu. Giá trị Sig. của kiểm định Bartlett bằng 0.000 , do đó các biến quan sát có tương quan với nhau xét trên tương quan tổng thể. Tại điểm dừng có Eigenvalue = 1.488 có 3 nhân tố được trích, phương sai trích đạt $59.555 \%$ thể hiện rằng 3 nhân tố được rút trích này giải thích được gần $60 \%$ sự biến thiên của dữ liệu. Tuy nhiên nhân tố "Thu nhận tri thức - KC" có hệ số tải nhân tố của biến $\mathrm{KC} 1$ nhỏ hơn 0.5 nên không đạt yêu cầu. Vì vậy, nhóm tác giả thực hiện phân tích EFA lần 2 cho nhóm biến $\mathrm{KD}, \mathrm{KC}$ và JS khi loại biến $\mathrm{KC1}$.

\section{Kết quả phân tích EFA lần 2 cho các biến DK, CK và JS}

Hệ số $\mathrm{KMO}=0.906>0.6$ nên EFA phù hợp với dữ liệu. Giá trị Sig. của kiểm định Bartlett là 0.000 , do đó các biến quan sát có tương quan với nhau xét trên tương quan tổng thể. Tại điểm dừng có Eigenvalue $=1.483$ có 3 nhân tố được rút trích, phương sai trích đạt $62.316 \%$ thể hiện rằng 3 nhân tố được rút trích này giải thích được gần $62,32 \%$ sự biến thiên của dữ liệu. 
Các thang đo trong 3 nhân tố đều có hệ số tải nhân tố lớn hơn 0.5 và hiệu của hệ số tải nhân tố giữa hai item của các biến đều lớn hơn 0.3 ; vì vậy đạt yêu cầu.

\section{Kết quả phân tích EFA cho biến phụ thuộc}

Kết quả phân tích cho thấy có 1 nhân tố được trích, tất cả các biến đều có hệ số tải nhân tố lớn hơn 0.5 , Eigenvalue đạt 3.125, lớn hơn 1; phương sai trích đạt 8.134 (lớn hơn 50). Hệ số KMO đạt 0.83 (lớn hơn 0.5 và nhỏ hơn 1 ). Kiểm định Bartlett's Test có sig bằng 0.000 (nhỏ hơn 0.05 ). Các chỉ số này đều đạt yêu cầu nên giữ lại tất cả các biến quan sát trong thang đo biến phụ thuộc.

\subsection{Phân tích nhân tố khẳng định CFA}

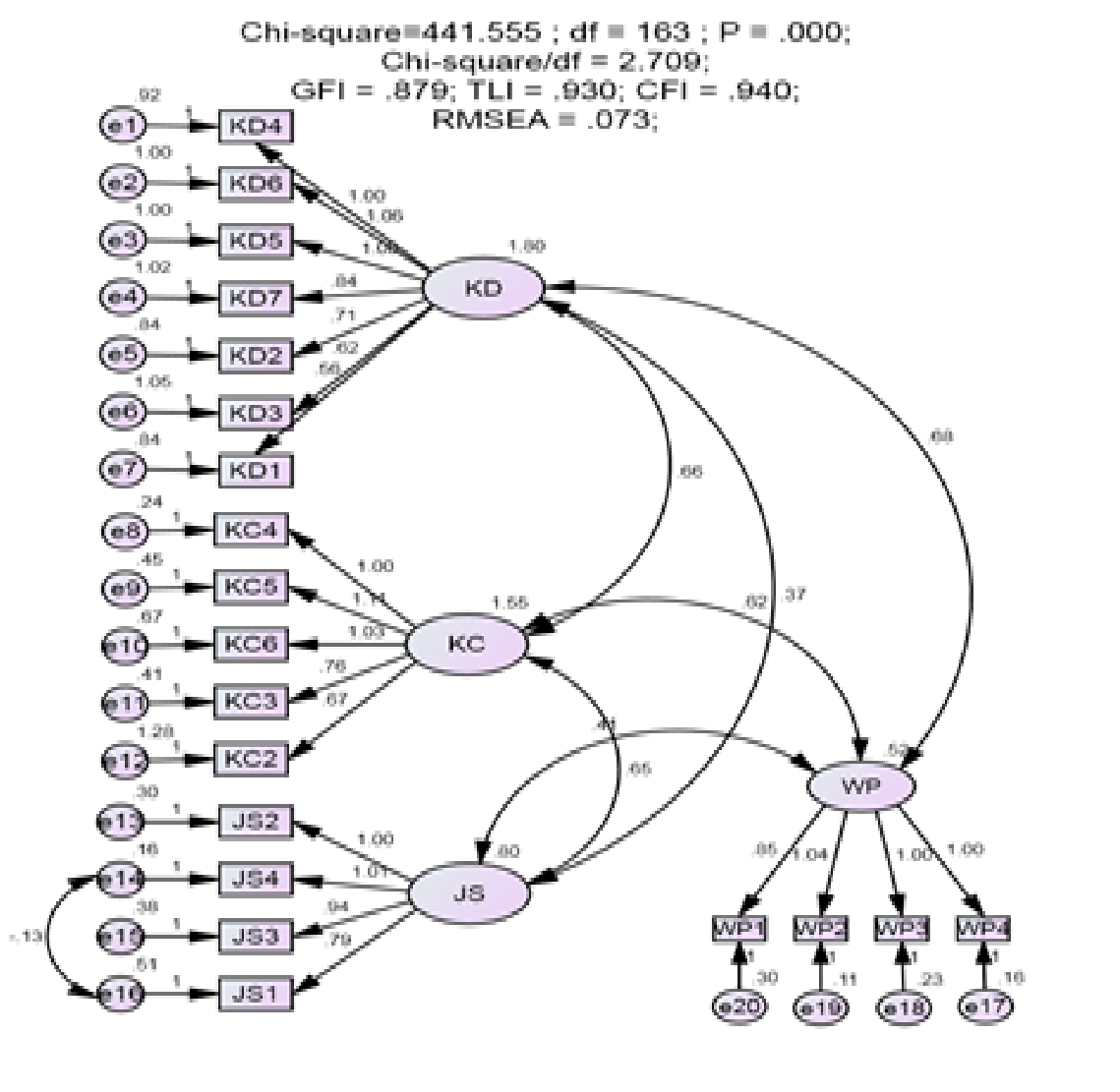

Hình 2. Kết quả phân tích CFA cho mô hình tới hạn

Nguồn: Kết quả phân tích dữ liệu của nhóm tác giả

Mức độ phù hợp chung: Phân tích CFA cho kết quả mô hình có giá trị thống kê Chi bình phương là 441.555 với 163 bậc tự do, giá trị $\mathrm{P}=0.000, \mathrm{CMIN} / \mathrm{df}=2.517<5$, đạt yêu cầu về độ tương thích. Các chỉ tiêu TLI $=0.930>0.9, \mathrm{CFI}=0.940>0.9$ và $\mathrm{RMSEA}=0.073<0.08$ đều đạt yêu cầu. Vì vậy có thể kết luận: Mô hình tới hạn đạt được độ tương thích với dữ liệu thị trường. Bên cạnh đó, trong phân tích CFA cần xem xét các chỉ số sau:

Giá trị hội tụ: Các trọng số đã chuẩn hóa đều $>0.5$ nên thang đo đạt giá trị hội tụ. 
Giá trị phân biệt: Các giá trị $\mathrm{P}$-value đều <0.05 nên hệ số tương quan của từng cặp khái niệm khác biệt so với 1 ở độ tin cậy 95\%. Do đó, các khái niệm nghiên cứu đều đạt được giá trị phân biệt.

Độ tin cậy: Độ tin cậy của thang đo được đánh giá thông qua hệ số Cronbach's Alpha, hệ số tin cậy tổng hợp (Composite Reliability) hoặc tổng phương sai trích (Average Variance Extracted).

\section{Bảng 2}

Tóm tắt kết quả kiểm định độ tin cậy của thang đo

\begin{tabular}{|c|c|c|c|c|}
\hline \multirow[b]{2}{*}{ Thang do } & \multicolumn{2}{|c|}{ Độ tin cậy } & \multirow{2}{*}{$\begin{array}{c}\text { Phương sai } \\
\text { trích }\end{array}$} & \multirow[b]{2}{*}{ Kết luận } \\
\hline & $\begin{array}{c}\text { Cronbach's } \\
\text { Alpha }\end{array}$ & Tổng họ̣p & & \\
\hline Cho, tặng tri thức - KD & 0.892 & 0.895 & 0.550 & \multirow{4}{*}{$\begin{array}{c}\text { Đạt yêu } \\
\text { cầu }\end{array}$} \\
\hline Thu nhận tri thức - KC & 0.876 & 0.914 & 0.685 & \\
\hline Sự hài lòng trong công việc - JS & 0.880 & 0.893 & 0.717 & \\
\hline Hiệu suất công việc - WP & 0.906 & 0.901 & 0.711 & \\
\hline
\end{tabular}

Nguồn: Kết quả phân tích dữ liệu của nhóm tác giả (2017)

Các thang đo đều hệ số Cronbach's Alpha lớn hơn 0.6 và nhỏ hơn 0.95 , độ tin cậy tổng hợp và phương sai trích đều lớn hơn 0.5 nên cả 4 thang đo đều đạt yêu cầu về độ tin cậy. Dữ liệu phù hợp để được đưa vào phân tích SEM tiếp theo.

\section{4. Đánh giá sụ phù hợp của mô hình bằng mô hình cấu trúc tuyến tính SEM}

Mô hình có giá trị thống kê Chi-bình phương bằng 487.519 với 164 bậc tự do, giá trị $\mathrm{P}$ $=0.000$; giá trị CMIN/df = $2.973<5$; đạt yêu cầu cho độ tương thích. TLI $=0.920>0.9, \mathrm{CFI}$ $=0.931>0.9$, RMSEA $=0.078<0.08$ đạt yêu cầu. Vì vậy có thể kết luận mô hình phù hợp với dữ liệu thị trường. 


$$
\begin{gathered}
\text { Chi-square }=487.519 ; \mathrm{df}=164 ; \mathrm{P}=.000 ; \\
\text { Chi-square } / \mathrm{df}=2.973 ; \\
\mathrm{GFI}=.871 ; \mathrm{TLI}=.920 ; \mathrm{CFI}=.931 ; \\
\text { RMSEA }=.078 ;
\end{gathered}
$$

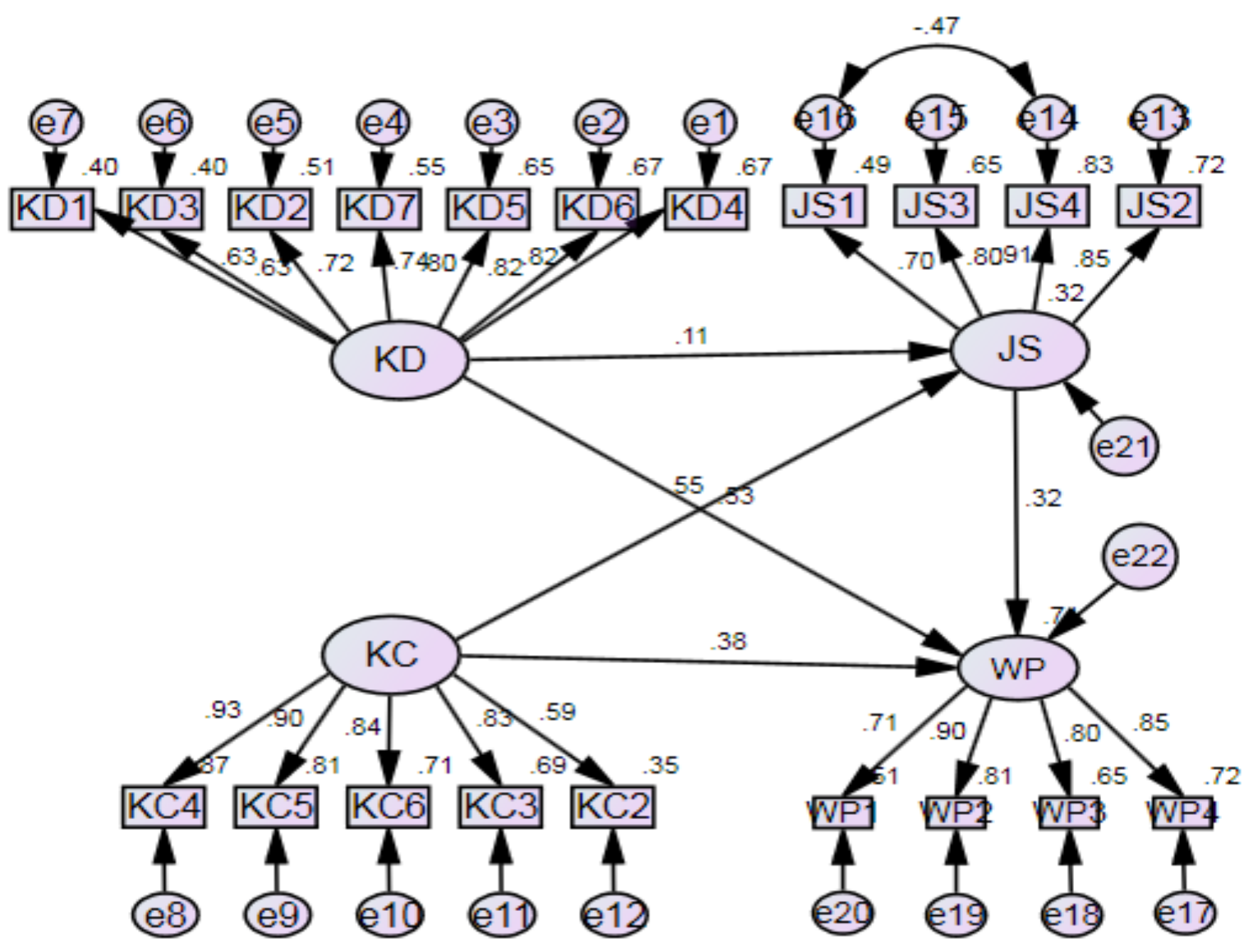

Hình 3. Kết quả SEM cho mô hình (đã chuẩn hóa)

Nguồn: Kết quả phân tích dữ liệu của nhóm tác giả (2017)

\subsection{Kiểm định giả thuyết}

\section{Bảng 3}

Kết quả kiểm định mối quan hệ nhân quả giữa các khái niệm nghiên cứu trong mô hình

\begin{tabular}{|c|ccc|c|c|c|c|c|l|}
\hline $\begin{array}{c}\text { Giả } \\
\text { thuyết }\end{array}$ & \multicolumn{2}{|c|}{ Mối quan hệ } & $\begin{array}{c}\text { Ước } \\
\text { lượng }\end{array}$ & $\begin{array}{c}\text { Ước lượng } \\
\text { (chuẩn hóa) }\end{array}$ & S.E. & C.R. & $\begin{array}{c}\text { P- } \\
\text { value }\end{array}$ & \\
\hline H1 & JS & $<---$ & KD & 0.072 & 0.111 & .034 & 2.143 & .032 & Chấp nhận \\
\hline H2 & JS & $<---$ & KC & 0.387 & 0.551 & .039 & 9.933 & $* * *$ & Chấp nhận \\
\hline H3 & WP & $<---$ & KD & 0.258 & 0.534 & .023 & 11.233 & $* * *$ & Chấp nhận \\
\hline H4 & WP & $<---$ & KC & 0.199 & 0.380 & .026 & 7.701 & $* * *$ & Chấp nhận \\
\hline
\end{tabular}




\begin{tabular}{|c|c|c|c|c|c|c|c|}
\hline $\begin{array}{l}\text { Giả } \\
\text { thuyết }\end{array}$ & Mối quan hệ & $\begin{array}{l}\text { Ước } \\
\text { lượng }\end{array}$ & $\begin{array}{l}\text { U’ớc lượng } \\
\text { (chuẩn hóa) }\end{array}$ & S.E. & C.R. & $\begin{array}{c}\text { P- } \\
\text { value }\end{array}$ & \\
\hline H5 & WP <--- JS & 0.239 & 0.322 & .037 & 6.437 & $* * *$ & Chấp nhận \\
\hline
\end{tabular}

Nguồn: Kết quả phân tích dữ liệu của nhóm tác giả (2017)

Giá trị $\mathrm{P}$ - value của các giả thuyết đều nhỏ hơn 0.05 ; hệ số ước lượng chưa chuẩn hóa và chuẩn hóa đều dương nên tất cả các giả thuyết đều được chấp nhận. Cả " $C h o$, tặng tri thức - KD" và "Thu nhận tri thức - KC" đều có tác động tích cực đến "Sụ hài lòng trong công việc $J S$ " và "Hiệu suất làm việc - WP" của nhân viên; "Sục hài lòng trong công việc - JS" cũng có tác động tích cực đến "Hiệu suất làm việc - WP".

\section{Bình giải}

Cho, tặng tri thức - KD có tác động trực tiếp đến sự hài lòng trong công việc và hiệu suất làm việc với hệ số ước lượng (đã chuẩn hóa) lần lượt bằng 0.111 và 0.534 . Nghĩa là, trong điều kiện các yếu tố khác không đổi, khi việc cho tặng tri thức của nhân viên tăng 1 đơn vị thì sự hài lòng của họ tăng thêm 0.111 đơn vị và hiệu suất làm việc sẽ tăng 0.534 đơn vị. Điều này cho thấy rằng, nếu kinh nghiệm làm việc và kiến thức chuyên môn càng được nhân viên chia sẻ thì công việc càng đạt hiệu quả cao. Kết quả này tương đồng với kết quả nghiên cứu của Javadi, Zadeh, Zandi, và Yavarian (2012). Theo Javadi và cộng sự (2012), việc chia sẻ tri thức không những góp phần cải thiện mối quan hệ giữa nhân viên và quản lý mà còn làm tăng hiệu quả làm việc và tăng năng suất sản xuất. Bên cạnh đó, theo Bảng 3 , giả thuyết “Cho, tặng tri thức có tác động tích cực đến sụ hài lòng trong công việc của nhân viên" có giá trị sig. cao nhất (gần bằng 0.05 ) và mức độ tác động lại yếu nhất bằng 0.111 ; chứng tỏ việc cho, tặng tri thức chỉ giải thích được gần 11 độ biến thiên của sự hài lòng trong công việc. Điều này phù hợp với thực tế vì không phải nhân viên nào cũng sẵn lòng chỉ dạy kinh nghiệm, kiến thức của mình cho đồng nghiệp. Chỉ những người có tính cách rộng mở, có thói quen và sở thích chia sẻ hoặc những người nhận thức được lợi ích của việc cho tặng tri thức thì mới cảm thấy vui vẻ, hài lòng khi chia sẻ với người khác.

Tương tự, “Thu nhận tri thức - $K C$ ” cũng có tác động trực tiếp đến sự hài lòng trong công việc và hiệu suất làm việc với hệ số ước lượng (đã chuẩn hóa) lần lượt là 0.551 và 0.380 . Khi các điều kiện khác không đổi, nếu việc thu nhận tri thức tăng thêm 1 đơn vị sự hài lòng tăng thêm 0.551 đơn vị và hiệu suất công việc cũng tăng thêm 0.380 đơn vị. Thực tế cho thấy, nhiều cá nhân đồng ý gia nhập một tổ chức hay doanh nghiệp nào đó không đơn thuần chỉ vì mức lương được đề xuất mà chủ yếu là vì dự đoán về những kiến thức họ sẽ nhận được trong tương lai. Vì vậy, việc thu nhận tri thức ảnh hưởng mạnh đến sự hài lòng của họ. Kết quả này tương đồng với quan điểm của Trivellas và cộng sự (2015), nhóm tác giả giải thích rằng hành vi cho và nhận tri thức sẽ làm nhân viên hài lòng hơn với công việc vì nó sẽ giúp phát triển năng lực cá nhân như: nâng cao sự sáng tạo, mở rộng các mối quan hệ và tăng kỹ năng làm việc nhóm.

Kết quả nghiên cứu cũng chỉ ra rằng, bên cạnh tác động trực tiếp thì “ $C h o$, tặng tri thức - KD" và “Thu nhận tri thức - KC" còn có tác động gián tiếp đến hiệu suất làm việc của nhân 
viên thông qua sự hài lòng của họ. Hệ số ước lượng về mức độ tác động này lần lượt là $0.059^{4}$ và $0.209^{5}$. Theo chỉ số này, việc tiếp thu kiến thức từ đồng nghiệp là yếu tố quan trọng giúp nhân viên nâng cao hiệu quả làm việc. Vì vậy, để đạt được thành tích lao động tốt và có nhiều cơ hội thăng tiến, các cá nhân cần tích cực học hỏi, trau dồi cũng như trao đổi tri thức với người khác.

Nghiên cứu này góp phần kiểm định mối quan hệ giữa chia sẻ tri thức, sự hài lòng trong công việc và hiệu suất làm việc của nhân viên. Kết quả nghiên cứu sẽ giúp nhà quản trị các doanh nghiệp khởi nghiệp trong lĩnh vực du lịch có nhiều chính sách hiệu quả hơn nhằm thu hút và duy trì đội ngũ lao động giỏi; qua đó có thể giúp doanh nghiệp tăng lợi thế cạnh tranh và phát triển bền vững.

\section{Kết luận và hàm ý quản trị}

Theo kết quả nghiên cứu, sự hài lòng trong công việc và hiệu suất làm việc của nhân viên trong các công ty khởi nghiệp trong lĩnh vực du lịch bị ảnh hưởng bởi yếu tố chia sẻ tri thức. Nghĩa là việc cho, tặng và thu nhận tri thức sẽ giúp cho nhân viên cảm thấy hài lòng hơn với công việc và hiệu quả làm việc của họ cũng tăng lên. Vì là doanh nghiệp mới thành lập nên phần lớn các công ty khởi nghiệp đều đang trong quá trình hình thành văn hóa tổ chức và bước đầu xây dựng danh tiếng cũng như tạo dựng lòng tin với khách hàng; vì vậy, việc nhân viên trao đổi kinh nghiệm, kiến thức và hỗ trợ nhau giải quyết các vấn đề khó khăn là rất quan trọng. Do đó, để có thể tồn tại và phát triển bền vững thì nhà quản trị cần có nhiều chính sách hữu hiệu để nhân viên tích cực chia sẻ tri thức cho nhau.

Thi̛ nhất, cần động viên nhân viên chia sẻ tri thức nhiều hơn bằng nhiều cách khác nhau như tạo điều kiện để nhân viên ở các phòng ban có cơ hội hợp tác, học hỏi thông qua việc luân chuyển vị trí, tổ chức các khóa học nâng cao kiến thức hay những cuộc thi về chuyên môn. Bên cạnh đó các nhà quản lý cần cung cấp nhiều thông tin phản hồi có giá trị để nhân viên có thể hoàn thiện kiến thức của mình, để họ cảm thấy việc chia sẻ tri thức là cần thiết.

Thú hai, những phần thưởng mong đợi luôn là những lợi ích dễ thấy thúc đẩy sự chia sẻ tri thức của mọi người. Vì vậy để nhân viên tích cực hơn trong việc chia sẻ tri thức thì các doanh nghiệp khởi nghiệp cần có nhiều hơn nữa các chính sách khen thưởng thích hợp để khuyến khích hành động này. Nhất là những phần thưởng dễ dàng nhìn thấy và cảm nhận được như tăng lương, thưởng theo quý, theo tháng hay theo kết quả công việc.

Thư $b a$, cần quan tâm nhiều đến yếu tố phát triển cá nhân. Ví dụ như thông báo cho người tham gia góp ý kiến rằng kiến thức của họ tạo nên sự khác biệt cho dự án, cho kế hoạch hay cho tổ chức. Bên cạnh đó cần loại bỏ rào cản sợ hãi không dám chia sẻ vì "mất mặt" bằng cách tạo ra bầu không khí làm việc khoan dung, thân thiện và cởi mở. Nhà quản trị nên luôn tạo điều kiện để nhân viên học hỏi từ những sai lầm, tham gia vào công cuộc đổi mới và cùng công ty vượt qua khó khăn.

${ }^{4} 0.111 * 0.534=0.059274$

${ }^{5} 0.551 * 0.380=0.209380$ 


\section{Hạn chế và hướng nghiên cứu tiếp theo}

Do tính chất công việc của đối tượng tham gia trả lời bảng câu hỏi khảo sát và nhiều người trả lời bảng câu hỏi khảo sát cho rằng không mang lại lợi ích gì cho họ hay tính bảo mật của kết quả khảo sát sẽ ảnh hưởng đến công việc nên có nhiều cá nhân miễn cưỡng trả lời những câu hỏi đặt ra. Điều này ít nhiều sẽ ảnh hưởng đến kết quả nghiên cứu.

Nghiên cứu này chỉ được thực hiện tại Thành phố Hồ Chí Minh, do đó khả năng tổng quát hóa chưa cao. Hiện tại ngành du lịch nước ta đang rất phát triển nên có rất nhiều doanh nghiệp khởi nghiệp ra đời; vì vậy cần thực hiện nghiên cứu ở nhiều tỉnh thành để có thể so sánh kết quả nghiên cứu từ đó làm tiền đề cho việc đề xuất các chính sách nhân sự hợp lý hơn.

\section{Tài liệu tham khảo}

Adams, J. S. (1963). Toward and understanding of inequity. Journal of Abnormal and Social, Psychology, 67(5), 422-436.

Aksoy, Y., Ayranci, E., \& Gozukara, E., (2016). A research on the relationship between knowledge sharing and employee performance: The moderating role of unethical behaviors in organizational level. European Scientific Journal, 12(4), 1857-7881.

Alegre, J., Sengupta, K., \& Lapiedra, R. (2013). Knowledge management and innovation performance in a high-tech SMEs industry. International Small Business Journal, 31(4), 454-470.

Bontis, N., Richards, D., \& Serenko, A. (2011). Improving service delivery: Investigating the role of information sharing, job characteristics, and employee satisfaction. The Learning Organization, 18(3), 239-250.

Business Link. (2009). Importance of knowledge to a growing business. Retrieved May 10, 2017, from https://www.infoentrepreneurs.org/en/guides/importance-of-knowledge-to-agrowing-business/

Chính phủ. (2009). Nghị định số 56/2009/NĐ-CP của Chính phủ: Về trợ giúp phát triển doanh nghiệp nhỏ và vùa [Decree No. 56/2009 / ND-CP of the Government: On assistance to the development of small and medium enterprises]. Retrieved May 14, 2017, from http://www.chinhphu.vn/portal/page/portal/chinhphu/hethongvanban?class_id=1\&mode $=$ detail\&document_id $=88612$

Chiu, C., Hsu, M., \& Wang, E. (2006). Understanding knowledge sharing in virtual communities: An integration of social capital and social cognitive theories. Decision Support Systems, 42(3), 1872-1888. doi:10.1016/j.dss.2006.04.001

Chow, W. S., \& Chan, L. S. (2008). Social network, social trust and shared goals in organizational knowledge sharing. Information and Management, 45(7), 458-465. doi:10.1016/ j.im.2008.06.007

Dalkir, K. (2007). Knowledge management in theory and practice. Burlington, MA: Elsevier Butterworth- Heinemann. 
Doanh nhân Sài Gòn. (2017). 80\% startup Việt đều gặp 4 khó khăn này [80\% of Vietnamese startups have these 4 difficulties]. Retrieved May 11, 2017, from https://doanhnhansaigon.vn/khoi-nghiep/80-startup-viet-deu-gap-4-kho-khan-nay1076642.html

du Plessis, M. (2007). The role of knowledge management in innovation. Journal of Knowledge Management, 11(4), 20-29. doi:10.1108/13673270710762684

Gao, F., Li, M., \& Clarke, S. (2008). Knowledge, management, and knowledge management in business operations. Journal of Knowledge Management, 12(2), 3-17.

Gerbing, D. W., \& Anderson, J. C. (1988). An updated paradigm for scale development incorporating undimensional and its assessment. Journal of Marketing Research, 25(2), 186-192.

Hall, D. T., \& Goodale, J. G. (1986). Human resource management: Strategy, design and implement. Northbrook, Il: Scott, Foresmen Company.

Henttonen, K., Kianto, A., \& Ritala, P. (2016). Knowledge sharing and individual work performance: An empirical study of a public sector organisation. Journal of Knowledge Management, 20(4), 749-768, doi:10.1108/JKM-10-2015-0414.

Hoang Trong, \& Chu, N. M. N. (2008). Phân tích dũu liệu nghiên cưu với SPSS tập 1 và 2 [Analyze study data with SPSS volumes 1 and 2]. Ho Chi Minh, Vietnam: Nhà xuất bản Hồng Đức.

Hooff, B. V. D., \& Ridder, J. A. D. (2004). Knowledge sharing in context: The influence of organizational commitment, communication climate and CMC usage on knowledge sharing. Journal of Knowledge Management, 8(6), 117-30.

Hoppock, R. (1935). Job satisfaction. New York, NY: Harper and Row.

Javadi, M. H. M., Zadeh, N. D., Zandi, M., \& Yavarian, J. (2012). Effect of motivation and trust on knowledge sharing and effect of knowledge sharing on employee's performance. International Journal of Human Resource Studies, 2(1), 210-221.

Lance, V. (2016). Top 20 reasons why startups fail [Infographic]. Retrieved May 12, 2017, from https://www.forbes.com/sites/groupthink/2016/03/02/top-20-reasons-whystartups-fail-infographic/?sh=479bed583911

Liao, C., To, P. -L., \& Hsu, F. -C. (2013). Exploring knowledge sharing in virtual communities. Online Information Review, 37(6), 891-909.

Lin, H. F. (2007). Effects of extrinsic and intrinsic motivation on employee knowledge sharing intentions. Journal of Information Science, 33(2), 135-149.

Madden, T. J., Ellen, P. S., \& Ajzen, I. (1992). A comparison of the theory of planned behavior and the theory of reasoned action. Personality and Social Psychology Bulletin, 18(1), 39. doi:10.1177/0146167292181001 
Masydzulhak, Ali, H., \& Anggraeni, L. D. (2016). The influence of work motivation and job satisfaction on employee performance and organizational commitment satisfaction as an intervening variable in PT. Asian Isuzu casting center. Journal of Research in Business and Management, 4(10), 1-10.

Nguyen, D. K. (2009). Giáo trình thục hành mô hình cấu trúc tuyến tính SEM với phần mềm AMOS [Curriculum for practicing SEM linear structure model with AMOS software]. Retrieved May 10, 2017, from http://www.thuvientailieu.vn/tai-lieu/bai-giang-thuchanh-mo-hinh-cau-truc-tuyen-tinh-sem-voi-phan-mem-amos-phan-1-42118/

Nguyen, T. D. (2011). Phưong pháp nghiên cúu khoa học trong kinh doanh [Scientific research method in business]. Hanoi, Vietnam: NXB Lao động - Xã hội.

Nguyen, T. D., \& Nguyen, T. M. T. (2011). Nghiên cứu khoa học marketing - Úng dụng mô hình cấu trúc tuyến tính SEM [Marketing science research - Applying SEM linear structure model]. Hanoi, Vietnam: NXB Lao động.

Nonaka, I., von Krogh, G., \& Voelpel, S. C. (2006). Organizational knowledge creation theory: Evolutionary paths and future advances. Organization Studies, 27(8), 1179-1208. doi:10.1177/0170840606066312

Nooshinfard, F., \& Nemati-Anaraki, L. N. (2014). Success factors of inter-organizational knowledge sharing: A proposed framework. The Electronic Library, 32(2), 239-261.

Ollo-López, A., Bayo-Moriones, A., \& Larraza-Kintana, M. (2016). Disentangling the relationship between high-involvement-work-systems and job satisfaction. Employee Relations, 38(4), 620-642, doi:10.1108/ER-04-2015-0071

Orces, S. O. S. F., \& Kim, Y. (2005). Behavioral intention formation in knowledge sharing: Examining the role of extrinsic motivators, social-psychological forces, and organizational climate knowledge sharing: Examining the roles of extrinsic motivators. MIS Quartely, 29(1), 87-111. doi:10.2307/25148669

Oyemomi, O., Liu, S., Neaga, I., \& Alkhuraiji, A. (2016). How knowledge sharing and business process contribute to organizational performance: Using the fsQCA approach. Journal of Business Research, 69(11), 5222-5227. doi:10.1016/j.jbusres.2016.04.116

Porter, L. W., \& Lawler, E. E. III (1968). What job attitudes tell about motivation. Harvard Business Review, 46(1), 118-126. doi:10.1108/EL-02-2012-0023.

Saeed, M. S. (2016). The impact of job satisfaction and knowledge sharing on employee performance. Journal of Resources Development and Management, 21, 16-23.

Schermerhorn, J. R. (1989). Management for productivity. New York, NY: John Wiley \& Sons.

Suliman, A., \& Hosani, A. A. A. (2014). Job satisfaction and knowledge sharing: The case of the UAE. Business Management and Economics, 2(2), 24-33.

Tam Mai (2017). Nguyên nhân thật sụ khiến khởi nghiệp thất bại [The real cause of startup failure]. Retrieved May 13, 2017, from https://doanhnhansaigon.vn/khoi-nghiep/nguyennhan-that-su-khien-khoi-nghiep-that-bai-1076997.html 
Teh, P. L., \& Sun, H. (2012). Knowledge sharing, job attitudes and organisational citizenship behaviour. Industrial Management \& Data System, 112(1), 64-82.

Trivellas, P., Akrivouli, Z., Tsifora, E., \& Tsoutsa, P. (2015). The impact of knowledge sharing culture on job satisfaction in accounting firms. The mediating effect of general competencies. Rocedia Economics and Finance, 19, 238-247.

Weiss, H. M. (2002). Deconstructing job satisfaction: Separating evaluations, beliefs, and affective experiences. Human Resource Management Review, 12(2), 173-194. 